\title{
3D cephalometry and artificial intelligence
}

\author{
J. Faure' ${ }^{1}$, A. Oueiss' ${ }^{2}$, J. Treil', S. Chen ${ }^{4}$, V. Wong ${ }^{4}$, J.-M. Inglese ${ }^{4}$ \\ 1 DFO Specialist, University Lecturer and Hospital Practitioner, Private Practice \\ 2 DFO Assistant, Nice. DDS, Dip. Orthodontics (Toulouse III, Anthropobiology) \\ 3 Neuroradiologist (Pasteur Clinic, Toulouse) \\ ${ }^{4}$ Research and Development Department, Carestream Health Rochester NY 14608 USA
}

\section{SUMMARY}

Orthodontists today work more and more in a three-dimensional (3D) universe with cone-beam examinations occurring more frequently, now supplemented by digital prints and 3D portraits.

So far these documents are used primarily as esthetic imagery; superimposition techniques, issued from geometric morphometrics, allow a pseudoquantified approach.

The implementation of true cephalic biometrics requires consideration of the complete craniofacial set at different anatomical levels (alveolodental/basic bone/frame or overall architecture) and in three dimensions. It must lead to a quantified description of the anatomy, dysmorphism, and the necessary therapy to correct it.

A parametric approach is needed to choose the landmarking, the definition of the orthogonal reference, the definition, and selection of parameters.

Given the number of parameters required for a description without fault, the use of a simple tool with artificial intelligence is inevitable.

\section{KEYWORDS}

3D cephalometry, 3D biometrics, dental landmarks, bone markers, choice of parameters, artificial intelligence

\section{INTRODUCTION}

For many years now, orthodontists have been trying to achieve a three-dimensional (3D) approach to the craniofacial system. To this end, in the past they would take three conventional cephalometric photographs taken in three dimensions: the lateral incidence and profile cephalometry, the basal incidence and basal cephalometry, and the frontal incidence and frontal cephalometry.
The fundamental challenge was the ability to easily identify the anatomical landmarks on the three snapshots. Consequently, points $A$ and $B$ would be relatively discernible on the snapshot in the lateral view but impossible to identify in the basal and frontal images while the transverse position remained impossible to pinpoint.

Address for correspondence:

Jacques Faure

10, place Lannes - 32021 Auch - France

E-mail: faure_ja@club-internet.fr

Article received: 21-04-2016. Accepted for publication: 30-05-2016.

This is an Open Access article distributed under the terms of the Creative Commons Attribution License (http://creativecommons.org/licenses/by/4.0), which permits unrestricted use, distribution, and reproduction in any medium, provided the original work is properly cited. 
Within recent times, the advent of the scanner and cone-beam x-ray has brought the orthodontist into the 3D realm. Thereafter, other 3D tools appeared; these capture the dental arches and cutaneous envelope with remarkable precision and color rendering.

From then on, the manipulation of 3D images pervaded our daily work and now 3D cephalometry is continuously made available to us and can be used at the clinic.

Of course, all 3D input tools have easy-to-use measuring instruments. For example, a digital impression scanner always uses a tool that measures the distance between two points, you simply have to point at landmarks $P$ and $\mathrm{Q}$ and the software immediately displays the PQ measurement. Similar$l y$, there are instruments for measuring the angle formed by two intersecting lines, the angle formed by two planes, the cartesian coordinates, etc.

Most often, however, the use of 3D tools is limited to the identification of a particular anatomical element (an impacted tooth for example or odontoma).

Though rare, authors advocate using the unique measurements or pseudo-measurements which result from the techniques of superimposition. The difference between the individuals compared (for example the subject's measurements before treatment and after surgery) is expressed by a colorimetric scale.

Finally, we can find articles where effective measurements were achieved using the software tools of the scanner or cone-beam x-ray. For example, in a study on the skeletal effects of maxillary disjunction by Hyrax, authors
Luebbert et al. ${ }^{31}$ use the following distances:

- Between the apices of the central incisors, those of the vestibular roots of the first premolars, and those of the vestibulomesial roots of the first maxillary molars.

- Between the pulp chambers of these same teeth.

- Finally, between the maxillary external cortical walls at the apex of the vestibular and vestibulomesial roots of both the premolars and the first molars.

It is obvious that these measurements have no daily clinical use, these parameters were chosen with a research objective, without any diagnostic benefits. The "landmark" sites, particularly the external cortex of the alveolar bone close to the apex, have neither the physiological identity nor the anatomical specificity to permit a diagnostic analysis.

To date, a complete software that can be used daily at clinic, which can cover the entire craniofacial system in the three dimensions of space, including the alveolar, dental, basal, and architectural floors (or maxillofacial framework), has not yet been made available. Such a program will, of course, have to precisely describe the anatomy and therefore be able to quantify the dysmorphia to be used as a guide in the establishment of the treatment plan.

In the following sections, we will examine the current uses of 3D imaging for therapeutic purposes and discuss the constraints and requirements for a complete and coherent 3D cephalometry. 


\section{D IMAGING AT THE CLINIC: THE USE OF GEOMETRIC MORPHOMETRIC TOOLS}

The use of 3D imaging in standard or clinical research is currently giving way to the geometric morphometric tools of superimposition, either by the direct use of geometric morphological software, (Morphological, Rapid Form etc.) or by integrating analog modules into the orthodontic or surgical software.

\section{Using geometric morphometry: the Procustes superimposition}

Firstly, these tools are used when comparing two individuals: $A$ and $B$.

In conventional superimpositions, the user identifies an area that is assumed to be a stable, "recorded area" and thus visualizes the evolution (growth, therapeutic, maturation, etc.) of the areas that are modified in relation to this stable reference area. It is therefore an operator-dependent choice. With the Procustes superimposition, the software corrects the scale, orienting the two subjects by parallelizing the axes of inertia and superimposing as precisely as possible, the minimum distances between the homologous points of subjects $A$ and $B$, on the entire subject studied. This technique separates size effects from form effects and is non-operator dependent.

Superimposition with a supposedly stable recorded area, will better demonstrate the variation observed within range of this area rather than a generalized Procustes superimposition.

The choice between these two methods will of course depend on the following:
- The nature of the phenomenon studied. If the evolution is global, a growth process for example, the Procustes superimposition will be the desired tool. If, however, the evolution is well localized, for example a mandibular advancement surgery, a superimposition on the "recorded area" (in this case the ascending ramus posterior to the osteotomy), will be preferred.

- The isolable or "dissectable" nature of the area of interest (actual or virtual dissection).

- The existence and distribution of landmarks or superimposition zones, on the stable site (recorded area) and on the supposedly mobile site.

The boundary is not as rigid as it may seem. With the distribution of the inhomogeneous landmarks, Procustes superimposition will begin to favor the area of maximum landmark density, or recorded pseudo-zone.

Procustes superimposition has evolved conventional methods by facilitating superimposition on a stable, recorded area which is often sought after and is considered a recalibration. In a maxillofacial surgical study, an area of superimposition is often taken in the anterior region of the skull base and the tools used to assess the $A / B$ deviations, standard Procustes superimposition tools will demonstrate the difference with the skull base recorded and unchanged.

Oueiss ${ }^{20,36,37}$ recalibrated points $\mathrm{SO}$, IO, HM (table I), to study the asym metry. 
Table l: The 14 trigeminal points. The eight points of the Treil framework model of the skeletal framework or the global architecture: RHM, LHM, RSO, LSO, RIO, LIO, RM, LM. The landmarks of the maxillary or mandibular bases are represented by: RM, LM, RFM, LFM, UNP, LNP, RGP, LGP.

(The mental points are both frame and maxillary base landmarks.)

\begin{tabular}{|c|c|c|c|c|}
\hline & & Environment & Skeletal Landmarks & \\
\hline RHM & LHM & MHM & Hammer Head: right and left & Frame \\
\hline RSO & LSO & MSO & Supraorbital: right and left & Frame \\
\hline RIO & LIO & MIO & Infraorbital: right and left & Frame \\
\hline RM & LM & MM & Chin: right and left & Frame and base \\
\hline RFM & LFM & MFM & Mandibular foramen: right and left & Base \\
\hline UNP & LNP & MNP & Nasopalataline foramen: upper and lower & Base \\
\hline RGP & LGP & MGP & Greater palatine canal: right and left & Base \\
\hline
\end{tabular}

\section{Landmarking: what to superimpose on?}

The description of an object's anatomy is either represented by a diagram of characteristic points or landmarks, (each one assigned to its cartesian coordinates) or on the complete 3D capture of the surface or a specific portion of it. The $A / B$ comparison will involve the superimposition of twopoint diagrams or the direct superimposition of surfaces $A$ and $B$.

The choice of technique depends on the existence or absence of easily identifiable anatomical landmark points. In any case, the reproducibility and accuracy of the landmarks must first be assessed ${ }^{48,35,30}$. In the absence of characteristic points, it is possible to use meshing methods to create virtual benchmarks distributed homogenously on a common surface without any immediately identifiable anatomical detail (pseudo-landmarks or semi-landmarks, Bookstein ${ }^{5,6,7}$ ).

Moreover, whatever the mode of anatomical identification, (point diagram or direct surface capture) it is possible to achieve a global superimposition over the entire anatomical element studied, or to determine a stable area of superimposition "the recorded area."

\section{What type of 3D capture?}

\section{Dentoskeletal}

For a predominantly voluminal recording, the $R x$ method was previously used but now cone-beam CT scans are required.

\section{Dental occlusal}

To capture the surface of the arch, the intraoral scanner can be used to scan the arch very quickly, with 
remarkable color and texture precision. It also allows for a reliable recording of the occlusal ratios.

\section{Mucocutaneous}

There are several possible methods for capturing the 3D surface of the facial skin or mucosal coating:

- Photogrammetry, derived from the principles of stereographic photography, a technique widely used in cartography ${ }^{10,11}$

- The optical scanner ${ }^{10,11}$

- Structured light 3D scanners

- Radiographic techniques such as scanners and cone-beam computed tomography

- Or even MRI imaging, 3D ultrasound, and holography.

However, the methods that provide a complete recording of a digitized $3 D$ surface are preferable to photographic techniques. The file can, therefore, be combined with the dentoskeletal file delivered by cone-beam computed tomography, by a fusion method, which will eliminate the need for optical methods such as stereographic photography.

\section{Fusion}

The ideal solution to obtain complete patient files is to conduct a cone-beam examination, a digital impression (via an optical scanner), and a 3D facial photograph.

The 3D images from the last two examinations are then integrated and combined in the 3D maxillofacial reconstitution of the cone beam by "fusion" methods ${ }^{32}$. This will compensate for insufficient resolution and the lack of color for teeth and occlusions as well as for the face and the smile.

Together they provide a complete understanding of the evolution of the occlusion, cutaneous surface, and skeletal architecture in addition to their ratios. A superimposition on the skull base can be used to assess the cutaneous and occlusal changes in relation to the orthodontic procedures, therapeutic tooth displacements, and/or surgical displacements.

It is possible to only consider the cutaneous variations or even the isolated dental occlusal variations when superimposing on the cutaneous "shell" alone 26,27,40: the observer will not be able therefore to assess the causal role of the tooth and skeletal displacements in possible cutaneous improvement. Similarly, we can identify and study cutaneous, ethnic, and morphological differences without having the appropriate tools to decipher the dental or skeletal roles in causation.

\section{Which stable reference area or "recorded area" should be chosen?}

If we choose superimposition on a stable reference area there are inherent challenges.

\section{Superimposition on the bone structures}

To study the maxillofacial evolution, many authors use the cranial base, or more specifically its anterior part, as their area of superimposition. Cedivanes has defined a superimposition protocol. On two cranial base images, $A$ and $B$, arranged in the same virtual space, homologous landmarks are 
identified. The program then determines the displacement matrix superimposing A on B. A manual adjustment is necessary. The $A / B$ differences can be quantified in the orthonormal system defined by the benchmark diagram. The limits of this method are consistent with a system based on the comparison of two individuals. We can also perform a "recalibration" on the orbital zone ${ }^{36,20}$, characterized by points IO, SO, and HM (table I).

\section{Superimposition on the mucosal structures, the palatine rugae}

To analyze tooth displacements during therapy, we have superimposed the images on casts; the first set of images are two-dimensional (2D) occlusal photographic images ${ }^{13}$. Then 3D photographs are used (photogrammetry and stereographic photography), before an optical scanner is finally used

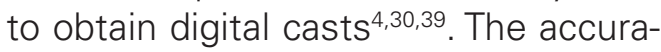
cy of the digital cast compared to the plaster mold is demonstrated. ${ }^{29,30}$ Digital casts prove to be extremely accurate for archiving and studying dental displacements. ${ }^{2,12}$

In the literature, most authors prefer superimposing on the anterior mucosal folds of the palate, the palatine rugae, and choose it as a reference area because it is a stable site $3,22,23,2,1,38$, but others maintain a more critical perspective. ${ }^{41,14}$.

\section{Superimposition of the cutaneous "shells"}

To study the evolution of the cutaneous "shell" different authors either advocate using a global superimposition or specific benchmarks. Kau and Richmond have identified five such benchmarks inclusive of the internal canthi of the eyes, commissures and nose tip. ${ }^{24-28}$ According to Moerenhout et al. there are 13: the sutures, wings, internal canthi $D$ and $G$, pupillaries $D$ and $G$, subnasal gnathion, zygions $D$ and $G$ and the right and left supraorbital points. ${ }^{33}$ Hoefert et al. have identified seven perinasal points. ${ }^{21}$

However, for these methods, the differences are generally color-coded and they are especially effective for A/B comparison.

Recently, a complex method developed by $\mathrm{Nanda}^{34}$ uses tools derived from neuroimaging: each subject's face benefits from a topological examination (spherical mapping). We can, therefore, record each facial shape and construct "an average face." The quantifiable diagnosis is the result of the "average" patient/subject superimposition which results in a color-coded visualization.

\section{Methods of assessing the localized A/B discrepancy}

These methods borrowed from geometric morphometry are ways of comparing two individuals with similar morphologies, or methods of comparing the same object at various stages of its evolution (growth, maturation, etc.) or at various stages of therapy (before, after treatment, after contention, relapse...)

These methods are aimed at measuring the difference which quantifies the pathology and the normalizing act of correction. They are essentially intended to assess the significance of the difference. They can be used for diagnostic purposes but only 
indirectly as this is not their primary purpose. Alternative methods include:

- Colorimetric assessment of the differences between surfaces $A$ and $B$, perpendicular to the surfaces (colorized visualization of the individual differences Rapid Form Plus Pack 2).

- Generalized Procustes Analysis (GPA, Dryden, and Mardia, 1993 ${ }^{15}$ )

- Thin-plate analysis (Bookstein7)

- Goodall test (Simple 3D)

- Wilk's lambda test (Morphologika ${ }^{2,1}$ )

- Principal Component Analysis (Jolife 1986, Dunteman, 1989)

- EDMA: Euclydian Distance Matrix Analysis.

For more details on these methods, the reader can consult the related studies 20,37 .

\section{Which subjects should be superimposed?}

The tools of geometric morphometry are designed to analyze slight differences between individuals of the same or similar types?

Their use in therapeutic follow-up, reviewing the therapeutic result, studying growth as well as the initiating and organizing therapeutic treatment, all correspond well to their capabilities. Though generally, the user tends to be more concerned with the significance of the change rather than the quantification of the measured difference.

The comparison of the same subject at stages T1 and T2 will reveal the subtlest changes.

However, the same is not true for its diagnostic uses:

- Subject A must be compared to a normomorphic subject to determine whether there is an active pathology.

- The difference must be quantified as a measure of the extent of the dysmorphia.

- The treatment is therefore conditioned by the superimposition.

A real normomorphic subject will be difficult to find and a virtual one will always be deprived of a little humanity! Virtual normomorphic subjects can be derived from the average morphology of a sample of normal subjects; which itself is not easy to find as there may be such challenges as: molar and canine class I; 2-mm incisal overlap or overhang, no missing teeth, no dentomaxillary disharmony $(\mathrm{DMD})^{24,27,28}$.

\section{CONSTRAINTS AND REQUIREMENTS FOR A TRUE THREE-DIMENSIONAL BIOMETRIC ANALYSIS}

After having seen the limitations of the tools of geometrical morphometry. What follows will consider the constraints imposed on the development of a true 3D biometric analysis.

As stated above, it must ensure that the entire craniofacial system is accounted for in the three dimensions of space, and at the three anatomical levels (alveolar, basal, and skeletal framework).

It should permit a quantified description of the dysmorphia, thereby allowing a quantification of the treatment plan.

It should of course focus on the interarch ratios, which is the key objective of any orthodontic therapeutic intervention. 
Table II: The five functional categories of parameters. MNP: middle of the anterior palatal canal, identified by its superior and inferior orifices (UNP and LNP). MHM, MFM, and MM: respective media of the hammerheads, HM, mandibular foramina, FM and chin, M, right and left. GIMY: y coordinate of the centers of inertia of the maxillary and mandibular incisors. RGP-LGP and RFM-LFM: distances between the greater palatine canals and mandibular foramina $D$ and G. TQIM: average torque of maxillary and mandibular incisive blocks.

\begin{tabular}{|c|c|c|c|}
\hline & Function & Example & \\
\hline 1 & $\begin{array}{l}\text { Position parameter of an area or } \\
\text { anatomical element in relation to } \mathrm{R}\end{array}$ & MNP y: & $\begin{array}{l}\text { Y coordinate of the mid-nasopalatine } \\
\text { canal }\end{array}$ \\
\hline 2 & $\begin{array}{l}\text { Morphological parameter of an } \\
\text { anatomical element }\end{array}$ & MHM-MM, MF-MM & $\begin{array}{l}\text { Overall mandibular length or } \\
\text { horizontal branch length }\end{array}$ \\
\hline 3 & $\begin{array}{l}\text { Parameter of the } \\
\text { high/low } \\
\text { relationship/discrepancy }\end{array}$ & GIMy-Gimy; RGP-LGP/RFM-LFM & $\begin{array}{l}\text { Posteroanterior discrepancy of } \\
\text { the upper and lower incisors } \\
\text { Ratio of the posterior widths of the } \\
\text { maxillary and mandibular bases. }\end{array}$ \\
\hline 4 & $\begin{array}{l}\text { High + low common pathology } \\
\text { parameter }\end{array}$ & $($ TqIM+Tqim)/2; $(\mathrm{GIMy}+$ Gimy)/2 & Angular and linear biproalveolar \\
\hline 5 & $\begin{array}{l}\text { High - low opposite pathology } \\
\text { parameter }\end{array}$ & TqIM-Tqim & $\begin{array}{l}\text { Class-II torque differential: } \\
\text { It accentuates or creates an overjet (or } \\
\text { limits the underjet in a class-III } \\
\text { situation) }\end{array}$ \\
\hline
\end{tabular}

We will successively consider landmarking, parameter selection, and artificial intelligence, all of which assist in accessing these data and in establishing a diagnosis and treatment plan.

\section{Landmarking and choosing frames of reference.}

\section{Skeletal Landmarks}

There are two main criteria for choosing skeletal landmarks:

- The chosen anatomical detail must play a major role in the growth and physiology of the area to be identified.
- The chosen anatomical detail must be identifiable without fail by any operator.

The osseous details (foramen) as they relate to the trigeminal system, are guides of maxillomandibular conjunctival growth and constitute a choice without any other alternative.

The 3D analysis program firstly relies on 14 points, which define the skeletal frame and mandibular base.

The eight points of the primary analysis of Treil highlight the bordering of the skeletal framework (Treil|42,43,44,46,47) and eight other points ensure the 
identification or location of the bases (Faure ${ }^{16-19}$ and Oueiss ${ }^{37}$ ). The chin points are mixed, they belong simultaneously to the framework and to the mandibular base. (Table I).

\section{Identifying teeth: the inertia matrix}

The mathematical identification of a solid object in space, as well as within the maxillomandibular assembly, is done by calculating the inertia matrix. This calculation defines the position of the center of inertia (Gx, Gy, and Gz) and its orientation by the data of the main axes of inertia. The use of inertia matrices is an "error-free" way of locating not only individual teeth but also groups of teeth which were joined or fused together during the orthodontic mechanical procedure (Treil J45).

\section{The futility of pure alveolar landmarks}

Odontologists are well aware that the alveolar bone is totally dependent on the teeth. In orthodontics, anteroposterior measurements are based on alveolar landmarks ( $A$ and $B$ for example: SNA, SNB, and ANB) show maximum correlations and quasi-identities, with measurements based on dental landmarks (centers of inertia of maxillary groups, GIM and mandibular incisors, Gim: GIMy, Gimy, and GIMyGimy); This makes any choice of alveolar landmarks superfluous, especially because the dental landmarks are more precisely identifiable.

\section{Choosing frames of reference}

Choosing a frame of reference is firstly a mathematical obligation, because the algebraic calculations must be based on quantified and signed variables. Thus, the parameters of the anteroposterior maxillomandibular ratios are signed distances (+ for class-III ratios).

The use of a direct, orthonormal trihedron associated with the anterior region of the skull region, is necessary to assess the maxillofacial morphology.

Alternatively, to locate the dental or paradental elements (reconstitution or fixed prosthesis), one must adopt the dentist's language: mesiodistal, vestibulolingual, and infraocclusion-supraocclusion; all of these refer to a system of curvilinear coordinates conforming to the shape of the arch.

\section{Critique of the benchmarks produced by $2 \mathrm{D}$ cephalometry}

The appeal of this new technique lies mainly in its ability to capture all the standard points of conventional cephalometry (A, B, pogonion, chin, etc.) and as such there is no "break" in the user's routine.

The method is simple:

- Identify a point on the 2D profile radiography obtained from $3 \mathrm{D}$ input, for example the right suborbital point.

- Then try to locate it in the right parasagittal plane on the 2D face-frontal X-ray obtained from the 3D input.

In addition to the severe inaccuracy, there is a major ethical problem herewhy must the patient be subjected to $3 \mathrm{D}$ capture only to derive clinical data that is of limited use, inaccurate, and 2D.

\section{The Parameters}

\section{Innumerable possibilities}

The first legitimate parameters are the coordinates of the 14 skeletal points ( $14 \times 3=42$ parameters). 
There are also many other parameters.

- There are (32) parameters for individual teeth and in terms of the groups: primarily 12 upper and lower incisors, two upper and lower molars, right and left, four upper and lower arches, two upper and lower right and left hemiarches, four of which can be located by three linear parameters and three angular parameters, which gives a grand total of 264 parameters.

- All the skeletal bipoints are facing point $\mathrm{C}_{14}{ }^{2}$, with each one having three linear and three angular parameters: 91 points.

- The triangular skeletal faces are at point $\mathrm{C}_{14}{ }^{3}$, with three linear and three angular parameters each: 364 faces.

- If we add the quadrangular faces (for example the lower mandibular face: RFM, LFM, RM, and LM), and if we introduce the interface or interpoint angles, we will quickly exceed one thousand parameters!

\section{The functional parametric classification}

Given the number of parameters likely to provide an effective description of the maxillofacial anatomy, parameter "recruitment" must be considered differently for individual cases, depending on the functional needs. To this end, a functional or categorical classification is required before the parameters are chosen.

- There are two functional categories: asymmetrical parameters and non-asymmetrical parameters.

- Technical categories related to the mode of measurement-these modes include:
1) Direction: anteroposterior, vertical, transverse: 3c

2) Pertinent/relevant anatomical element: alveolar/basal/skeletal framework or architecture: A, B, C: 3c

3) Location of the area of interest within the anatomical element studied or position of the landmarks: anterior/ middle/posterior: $3 \mathrm{c}$

4) Measurement technique: $5 c$

$\checkmark$ Direct measurement or function of several parameters (example: coordinate of the center of inertia of the incisor group or the maxillary $\operatorname{arch} . .$.

$\diamond$ The ratio of the linear (distance) and angular measurements: the first form requires a scale correction.

$\checkmark$ Distance or vector (scalar distance or signed value), scalar angle, or signed angle.

$\diamond$ Cartesian and curvilinear coordinates.

$\checkmark$ Direct or projected measurements (DEM for angles).

\section{- Function}

According to the parameter's function (position $\mathrm{R}$, morphology, $\mathrm{B} / \mathrm{H}$ ratio, $\mathrm{B} / \mathrm{H}$ pathological differential, common high/low pathology): 5c.

\section{- Category enumeration}

Non-asymmetry/asymmetry: 2c

Measurement mode (§ 1, 2, and 3), without considering the different technical possibilities of measurement (§4): $3 \times 3 \times 3=27 c$

Functions: $5 c$

A flawless description of the anatomy or maxillofacial pathology would necessitate as many as 270 parameters! 
That equates to 270 parameters if we only keep one parameter per category instead of proposing eight or 10 in each (§ 4).

\section{Note 1: concerning the types of parameters}

Among the types of parameters available to us, many are already present in the usual 2D analyses, for example:

- Position (R): is the position of an anatomical element or landmark/ skull base: SNA, SNB, 1/Frankfort

- B/H ratio: Adobos; ANB = SNA-SNB; - morphology, gonial angle.

On the other hand, two types of parameters are very effective and yet are only intuitively used without systematic "encryption."

- Common pathology high-low: (TqIM+Tqim)/2, angular biproalveolar.

B/H pathology differential: (TqIMTqim)/class-II incisal torque differential. It creates or accentuates the occlusal class II at the incisor level (or moderates class III in a class-III context: class-III compensation). A negative value corresponds to a class-III differential (or class-II compensation).

\section{Note 2: explanatory examples}

- Gimx is a position asymmetry parameter of an anatomical/transverse/ alveolar/anterior landmark element

The Gimx transverse coordinate (/R) of the center of inertia of the lower incisor group.

MNPy-MM is a non-asymmetry discrepancy-based parameter: high/low, anteroposterior/basal (basal bone)/ anterior landmarks.

MNPy is a posteroanterior coordinate of the middle of the anterior palatal canal (anterior maxillary basal landmarking).

$\mathrm{Mm}$ is a posteroanterior coordinate between the mental foramen (anterior mandibular basal landmarking).

MNPy-MMy: class-II discrepancy between the maxillary and mandibular bases, anterior segment.

\section{Selection}

A discriminant analysis or simple observation of the mean values of the parameters studied in the reference sample and in a pathological sample (t-test) makes it possible to identify the most effective parameters in the detection of major pathologies. Stringent selection should decrease the number of descriptive figures to approximately 100 while ensuring a precise description of the pathology.

For example, there are many measurements of the same type. Thus, from the posteroanterior position of the anterior basal mandibular region, the possibilities are as follows:

MMy coordinate (R), <MHM-MIO$\mathrm{MM}>$, <MHM-MSO-MM>, <MSO$\mathrm{MIO}-\mathrm{MM}>$, quadratic angles of the faces RSO-LSO-RIO-LIO/RIO-LIORM-LM, MHM-MM, MHM-MM/ MHM-MIO, MHM-MM all projected on Oy.

Only one is kept: MMy is the most statistically effective parameter for differentiating a class-II or class-III pathological group from the "normal » reference group. 


\section{Artificial Intelligence}

Despite the statistical sorting and the major elimination of many possible parameters, functional analysis has shown that it is impossible to decrease the number of descriptive figures (below 100) to 200.

This is hardly surprising because a conventional 2D analysis already contains about 20 measurements, including the anteroposterior orientation, the incisor alveolar level, and one or two vertical measurements. However, it does not account for basimaxillary or architectural landmarks; the transverse direction; or even transverse, vertical, or anteroposterior asymmetry.

Artificial intelligence is needed to synthesize and produce a "written" diagnosis.

This program must first approximate the neighboring parameters: molar discrepancy parameter (positive count for class II), and $\mathrm{B} / \mathrm{H}$ incisal discrepancy parameter (positive count in class II), and provide a comment.
For example, a general schematic of the class-III trend shows that the measured clinical situation is characterized by:

- a decreased overjet (underjet observed and measured); - molar ratios: (0) normal or neutral (measured class-I relation). The comment displayed must be "underjet despite class-I molar ratios."

Clearly, if with two neighboring parameters there are nine possible comments, then with three neighboring parameters there will be 27 possible comments, etc.

Artificial intelligence must be able to provide a final synthesis-e.g., "excess of the right hemiface" when there is a simultaneous right posteroanterior excess and a right vertical excess on the left lateral mandible.

To recognize a major syndrome, for example, this "excess of the right hemiface" defined above, the software must pair the skeletal parameters (of the frame or bone base) in the three dimensions of space.

\section{CLINICAL CASE COMPARING THE IMPLEMENTATION OF GEOMETRIC MORPHOMETRIC METHODS AND A RIGOROUS PARAMETRIC CEPHALOMETRY}

This patient came to our office having transferred from a nearby town where maxillary bands were placed without any mention as to the asymmetry diagnosis.

\section{Diagnosis}

Synthesized diagnosis: class-III hyperdivergence with excess asymmetry of the right hemiface. The diagnosis includes a small alveolar class III, a rotation of the mandibular arch to the left/maxillary (deviation from the centers and a more pronounced class III on the right), and a displacement to the left of the mandibular arch (slight right maxillary exoalveolar) (Figs. 1-4). 


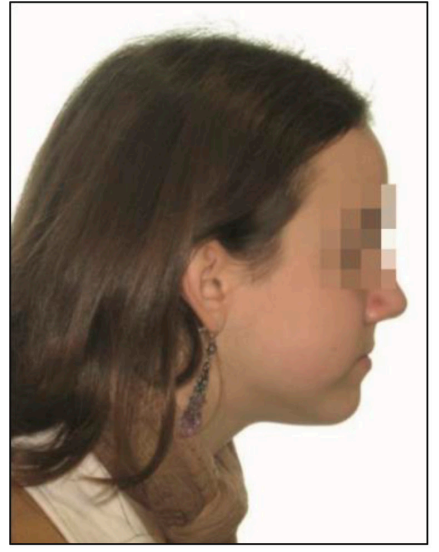

a

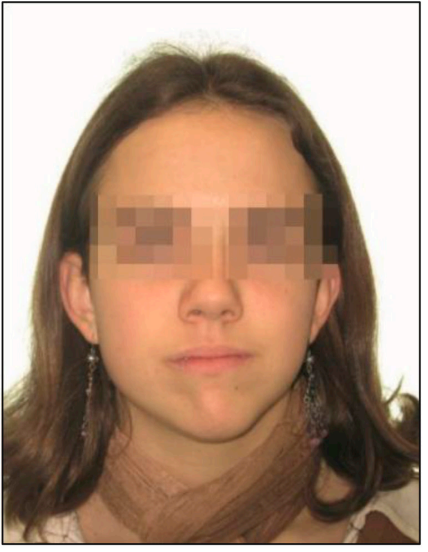

b

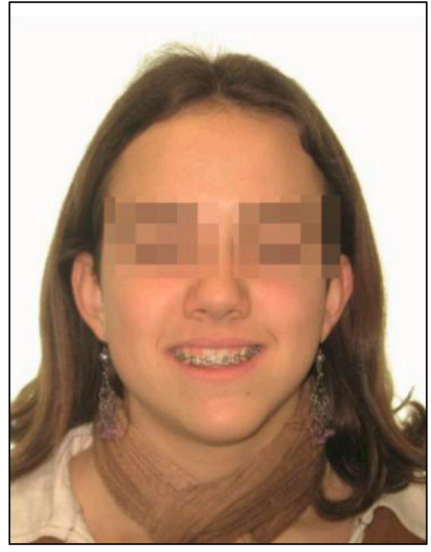

C

Figure $1 a, b, c$

Preliminary files. Portraits, profile, smiling face, face at rest (6/09; 15a 6m).

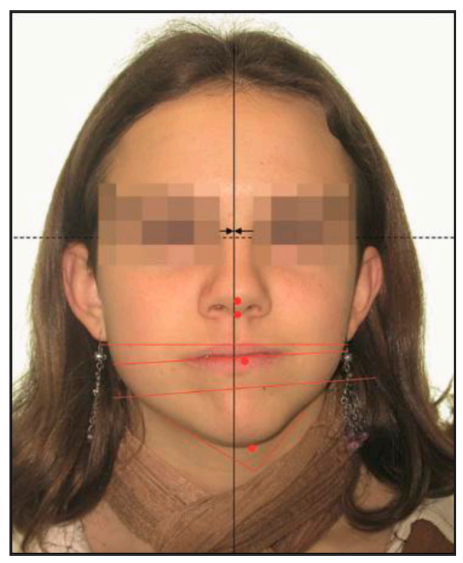

Figure 2

Portrait of the face at rest: facial lines (6/09; $15 a 6 m)$.

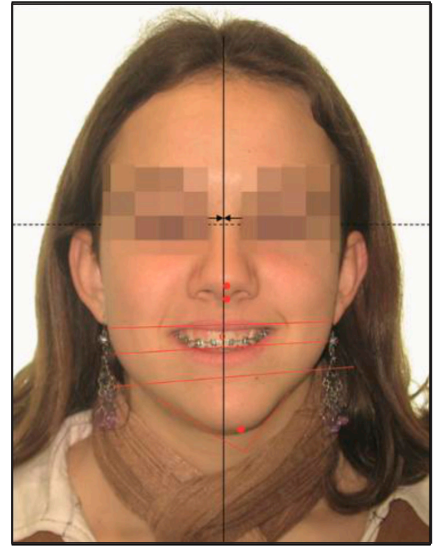

Figure 3

Portrait of the face at rest: facial lines (6/09; 15a 6m).

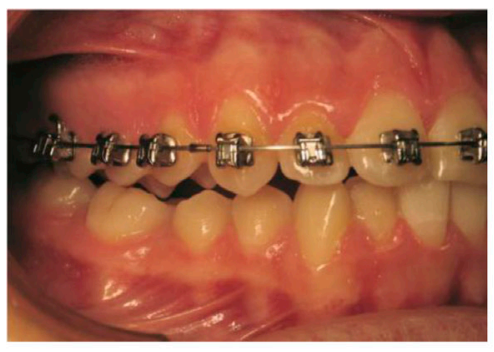

a

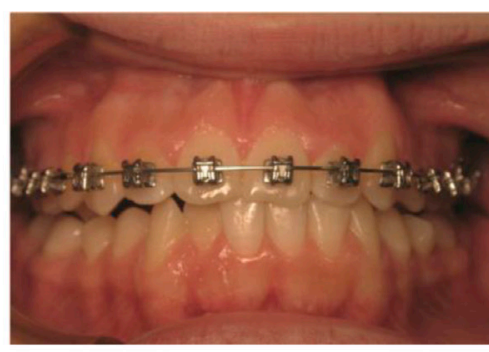

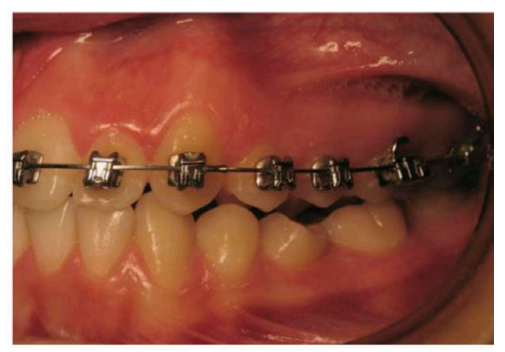

Figure 4

preliminary files. Right, frontal, and left intraoral views. (6/09; 15a 6m). 
There is a class-III skeletal pattern with a clear facial concavity; hyperdivergence is responsible for an hourly shift in the arches.

Facial asymmetry, caused by a right hemiface excess, is characterized by a maxillary deviation to the right and mandibular deviation to the left.

At the alveolar level, the left mandible experiences a characteristic deviation to the left of the mandibular arch and a deviation to the left of the mandibular interincisal median (rotation to the left of the mandibular arch in the horizontal plane.)
In addition, there is a "rolling" of the two arches, which is more significant in the lower arch, and affects the mandibular base. It is lower on the right compared to the left (vertical compensations are made by increasing the maxillary and mandibular alveolar processes to the right).

Lastly, maxillary and mandibular anterior and lateral class-III compensations and additional left lateralmandibular compensations are noted (Figs. 5, 6).

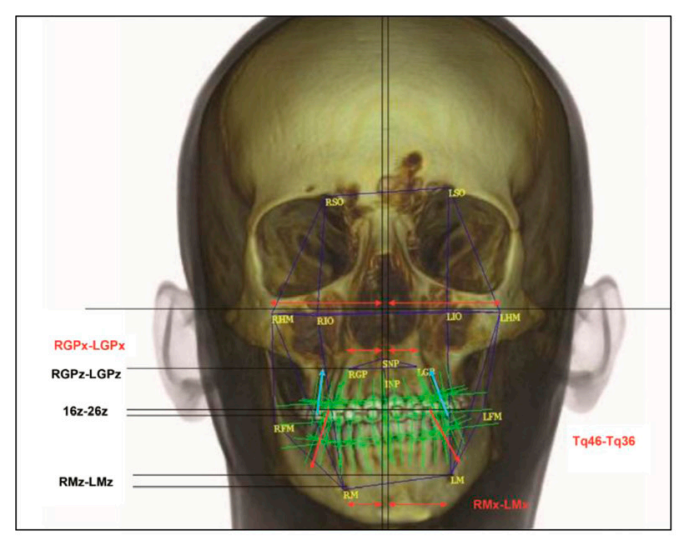

a
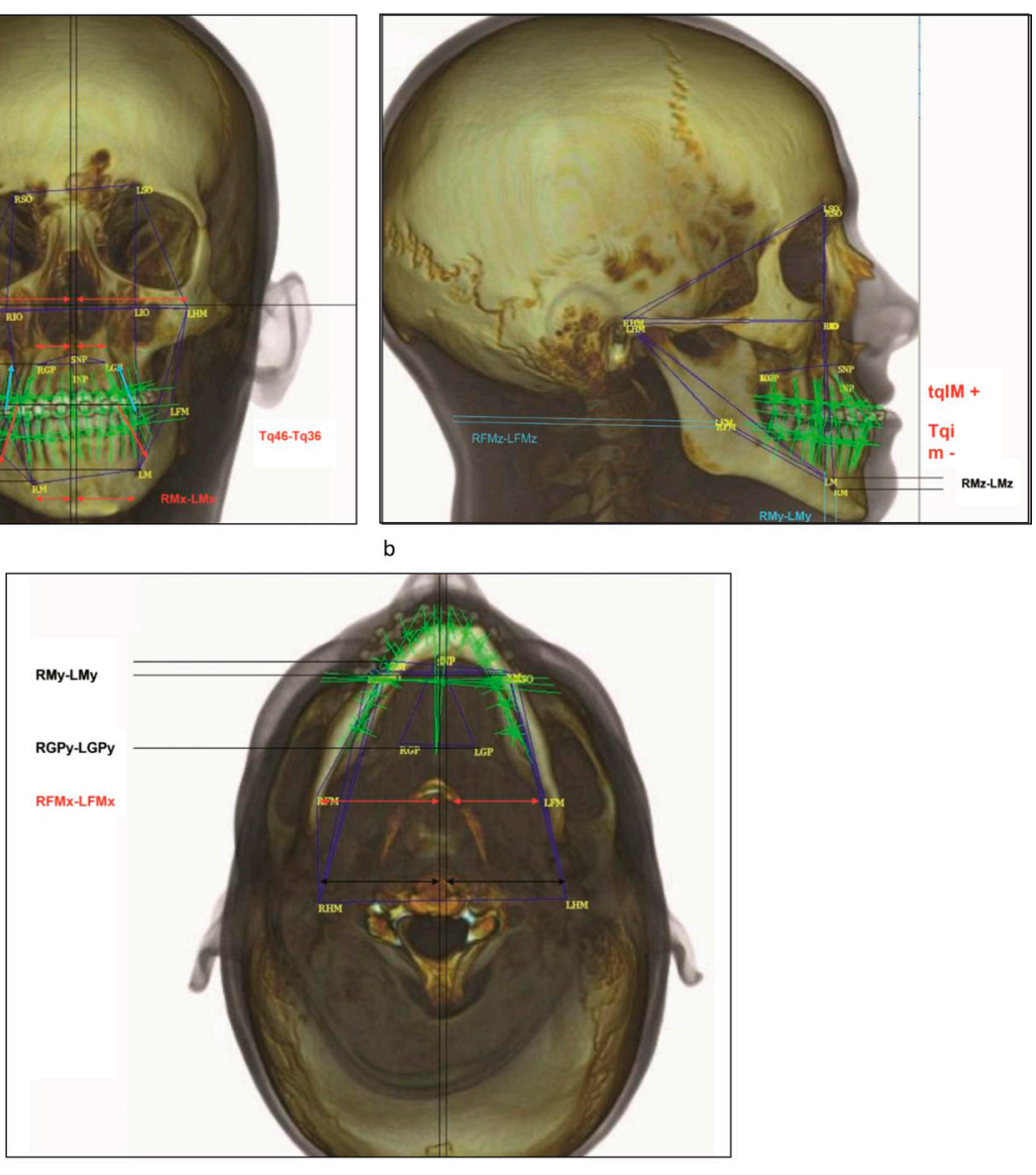

Figure 5

craniofacial scan number. Facial, profile, and basal views; with trigeminal benchmarks and the most significant asymmetry parameters. 


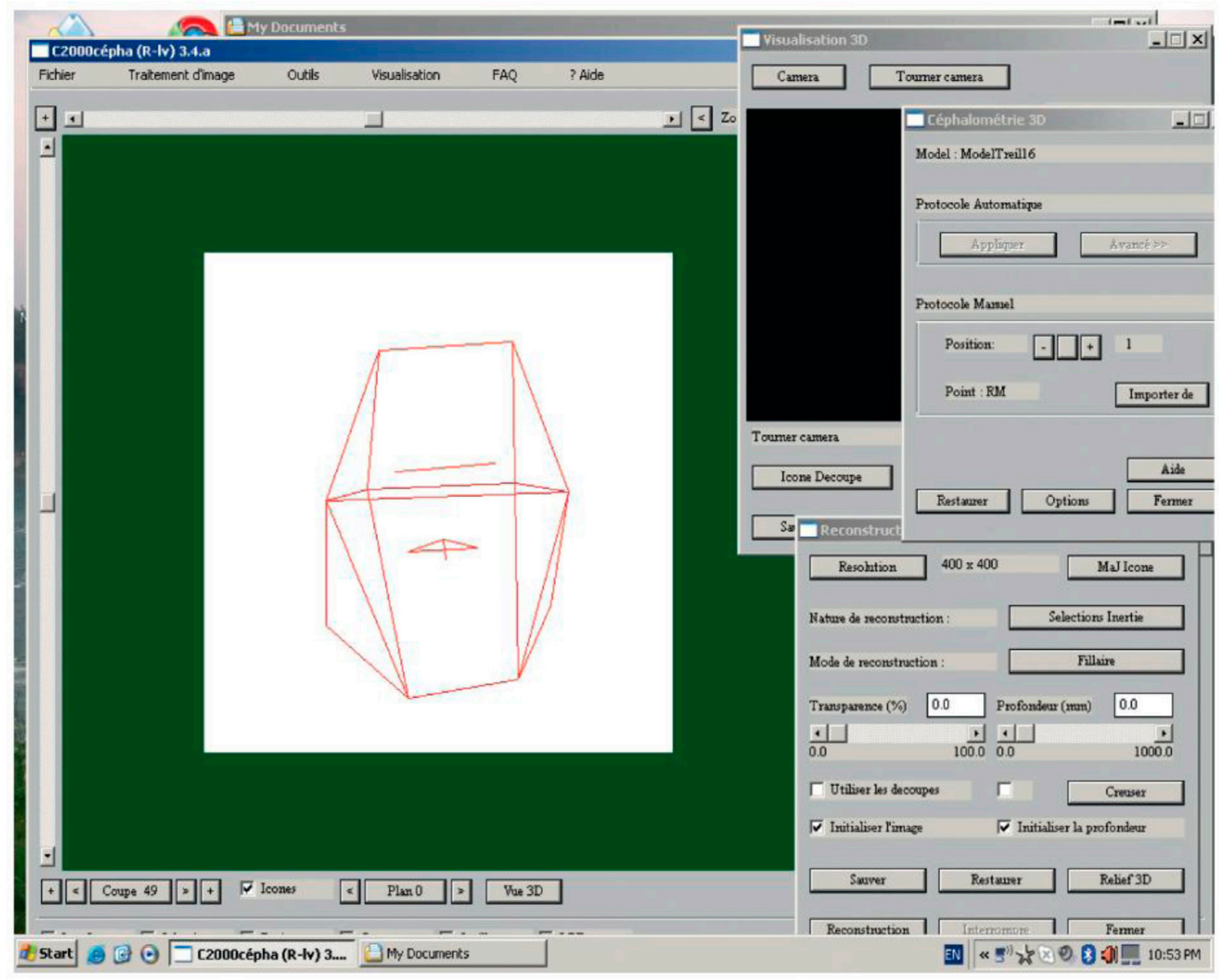

Figure 6

The 8 trigeminal points diagram of Treil showing the general maxillo facial fram. Here a case of asymmetry and class III.

\section{Treatment}

Treatment first involved a difficult removal of anteroposterior compensations and asymmetry compensations. The removal of the vertical compensations (right posterior lowering of the maxilla along the right unilateral vertical excess of the mandible has created a right posterior lateral infraocclusion; Figs. 7-9).

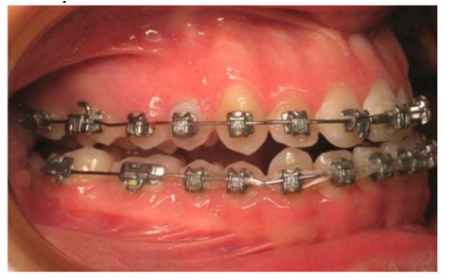

a

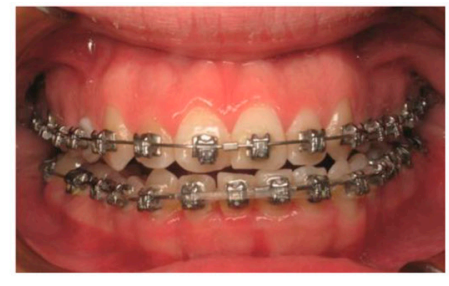

b

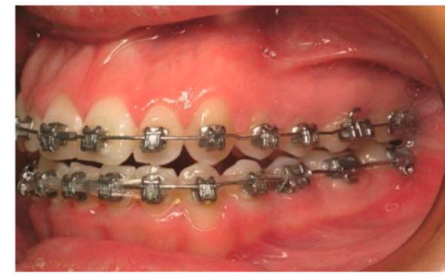

C

Figure $7 a, b, c$

Right, frontal, and left intraoral views: removal of compensations. (11/09; 15a 11m). 


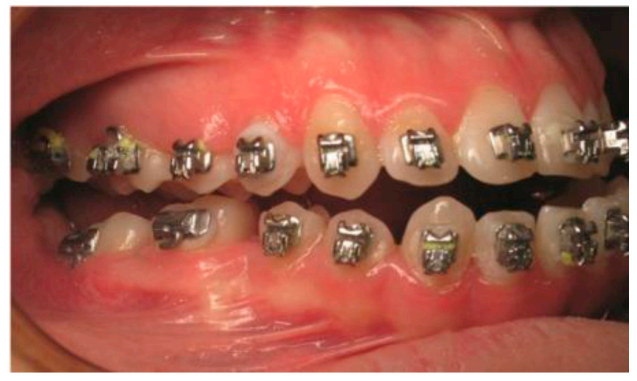

Figure 8

Intraoral view: vertical decompensation. (02/10; 16a $2 \mathrm{~m})$.
Surgery included maxillary advancement, a more significant mandibular advancement on the left with a mandibular inclination (elevation to the right of the mandible), and a genioplasty to reposition the chin (Figs. 10-13).

\section{Analysis of the dysmorphia}

An analysis of the dysmorphia was performed in 3D using the C2000 Cepha program, a parametric cephalometry

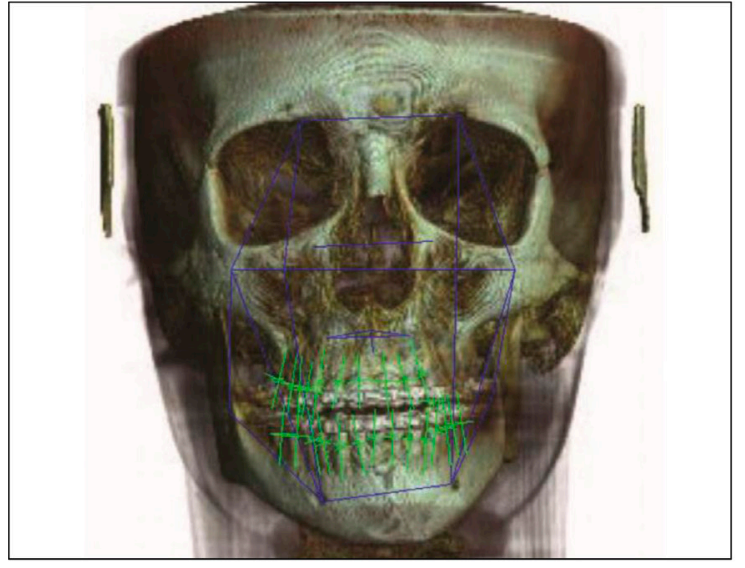

a

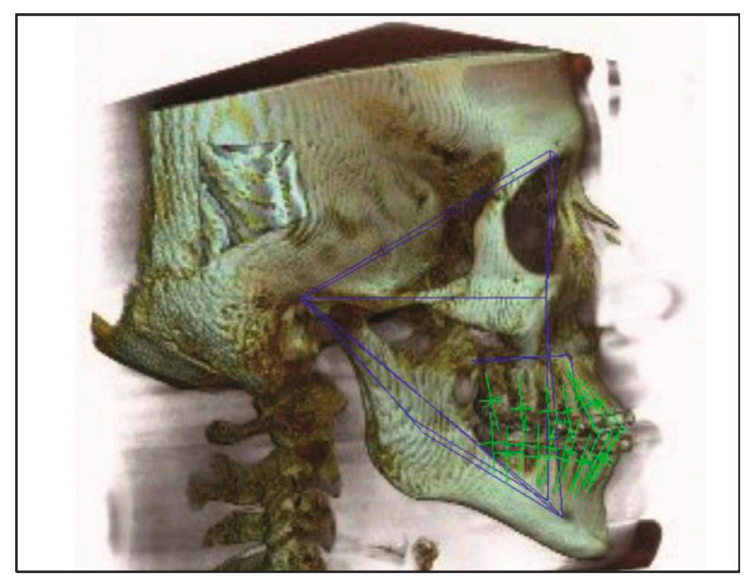

b

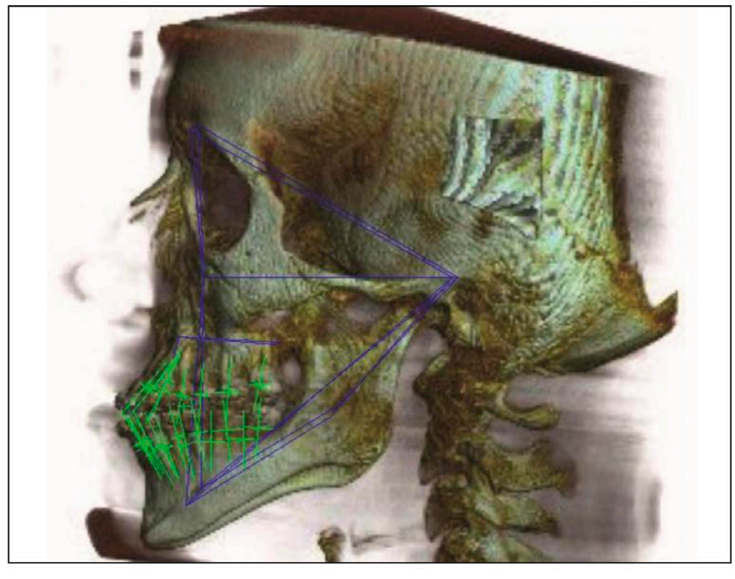

Figure $9 a, b, c$

Preoperative craniofacial scan no. 2; frontal v4iew of face and the right and left profiles. (06/10; 16 a 6m). 


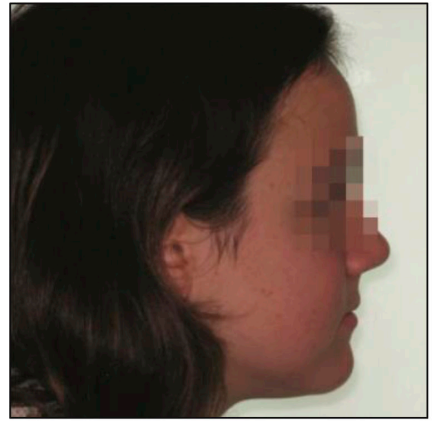

a

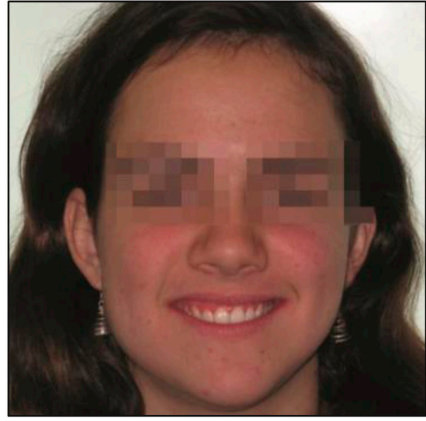

b

Figure 10a, b, c

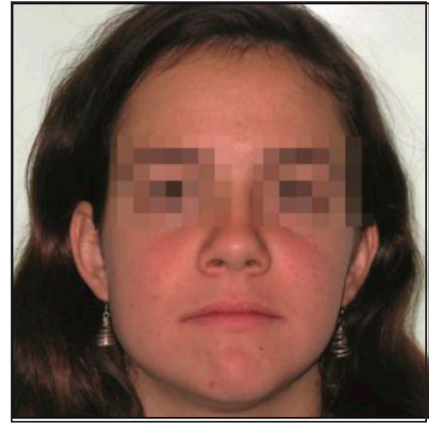

C

Pictures of the profile, smiling face, and the face at rest. Band removal. (12/10; age 17 years).

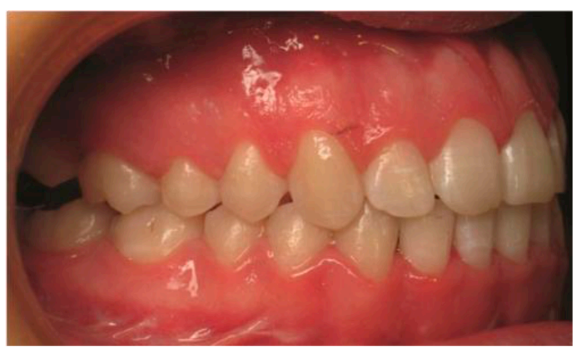

a

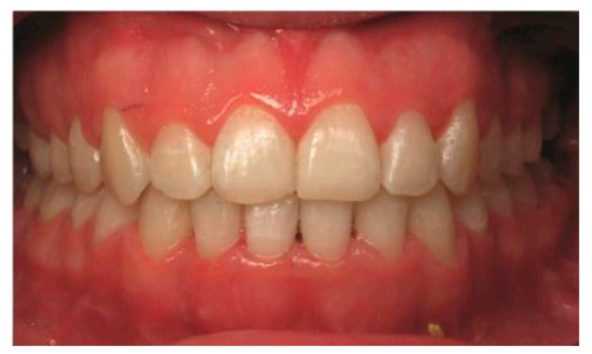

Figure $11 a, b, c$

b

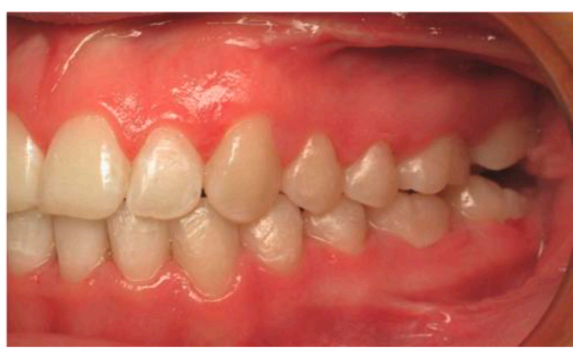

C

Right, frontal, and left intraoral views. Bands removed. (12/10; age 17 years).
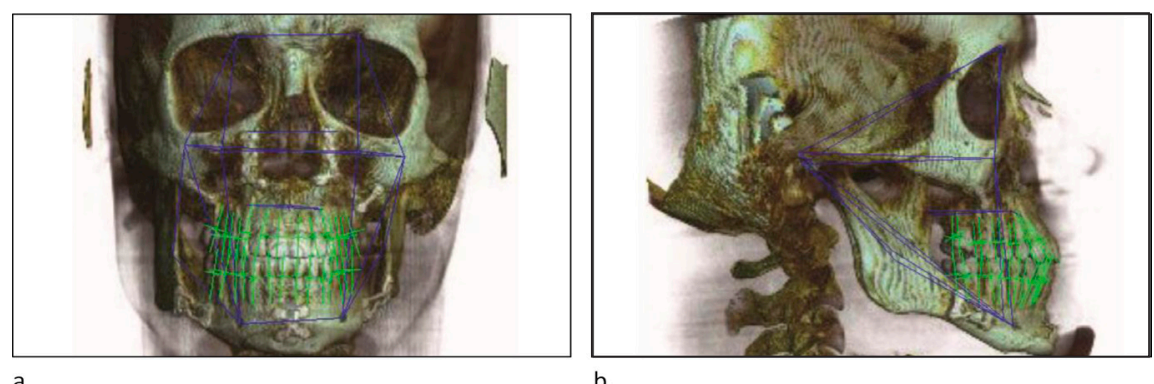

b

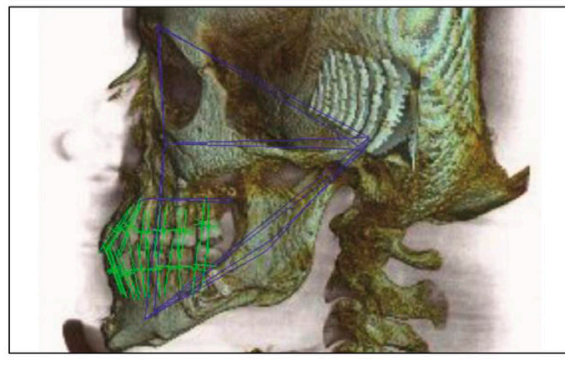

Figure $12 a, b, c$

Postoperative craniofacial scan no. 3. Frontal view of the face and the right and left profile views. Bands removed. (12/10; age 17 years). 


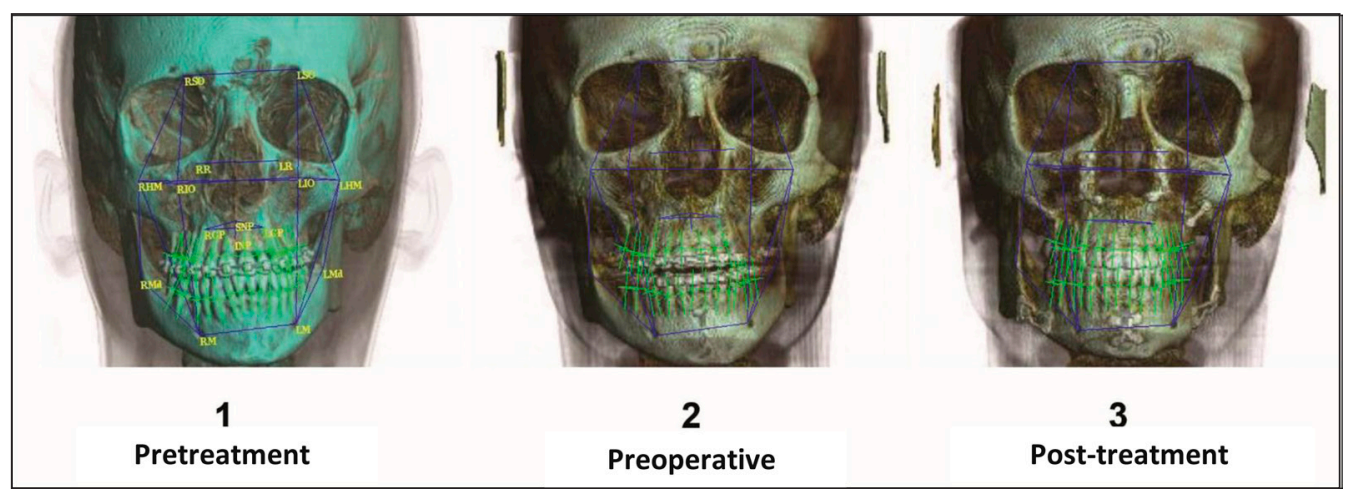

Figure 13

Comparison of frontal views: early multiband treatment: preoperative and postoperative.

program that we used at that time. The 14-point trigeminal pattern and dental landmarking, determined by the inertia matrix calculation, were already performed in the parametric analysis.

The use of geometric morphometric tools for superimposing on the orbital area (files 3/1, Rapid form, Fig. 14 or files 3/2, D1 mesh, Fig. 15) makes it difficult to (1) comprehend the complexity of the pathology and its surgical treatment, (2) "follow" the overall evolution or more specifically the final stage of the surgical evolution; color coding the variations facilitates our understanding.

On the other hand, using these tools for diagnosis is virtually impossible in this instance.

The ar eas of significant orthodontic or surgical displacement (red T3/blue T1, Fig. 14 Rapid form) can be seen.
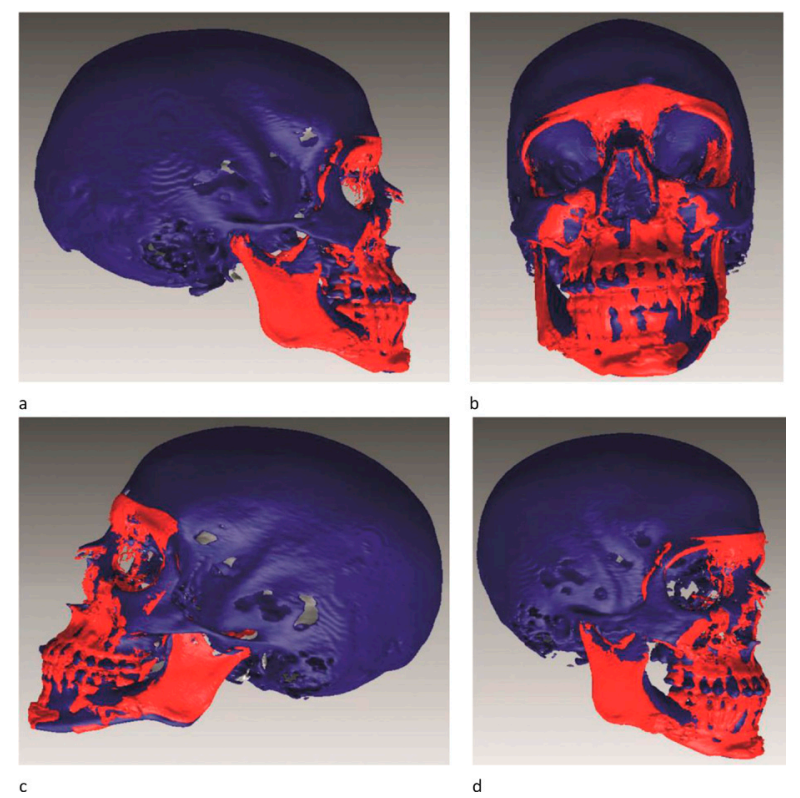

Figure $14 a, b, c, d$

Superimposition readjusted on the orbital sector (Rapid form): 1 = end, 3 = beginning. Right, frontal, left, and three-quarter views. 


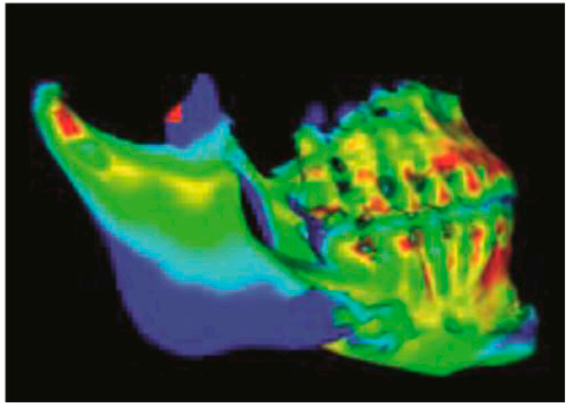

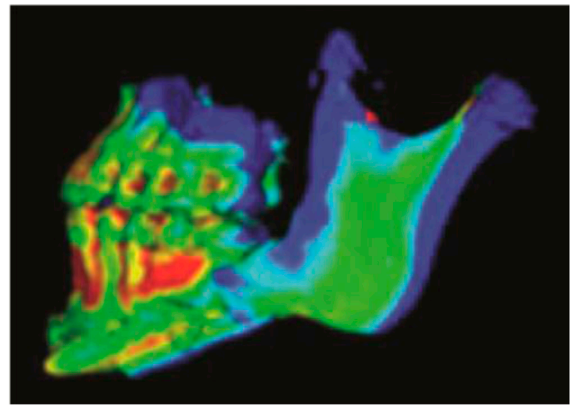

b

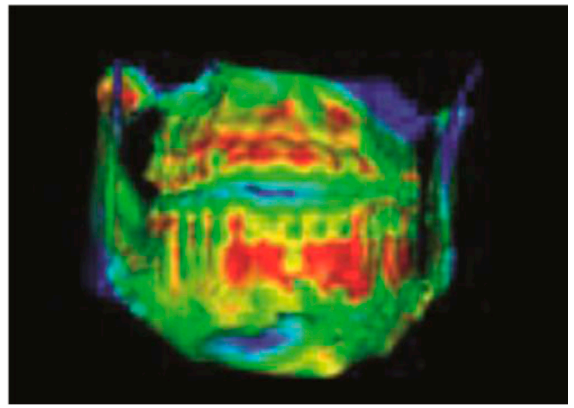

C

Figure 15a, b, c

Recalculation of superimposed reconstructions: right, front, and left. End of treatment n. 3 /end of treatment n. 2 (D/ mesh).

- Incisor modifications: removal of compensations (high recoil, low advancement) and a surgical advancement

\section{CONCLUSION}

A true 3D biometric analysis needs to be developed. As we have discussed above, there are rigorous and often contradictory constraints involved:

- The accurate identification of 3D landmarks based on a stable anatomical structure.

- Choosing numerous parameters to ensure a complete description.

- The fact that no more than 12 parameters are necessary to give the reader the ability to easily discern at a single glance.

- Significant parameters are easily comprehensible.

- Linear measurement parameters are preferred and should be parallel to the orthonormal axes.
- Chin modifications: repositioning genioplasty

- Gonial modifications: the left mandibular advancement slightly "pushes out" the right gonial angle.

- Choices made are based on the statistical analysis of the orthodontists' specific hierarchy.

- A selection of parameters allowing an accurate description of any dysmorphia.

An overview of the superimposition techniques most often used in a "pseudobiometric" analysis, shows the inadequacy of these tools and therefore the need for a parametric approach. However, the latter in turn requires the use of artificial intelligence which has the following capabilities:

- Synthesize and compare numerical values.

- Manage the large number of parameters. 
- Describe the dysmorphia in English not in figures.

- Only display the figures at the express request of the user.
Conflict of interest: JF, $A O$, and JT declare that they have no conflict of interest. SC, VW, and $J M I$ belong to the RD Department, Carestream Health Rochester.

\section{BIBLIOGRAPHY}

1. Almeida $\mathrm{MO}$, Phillips $\mathrm{C}$, Kula K, Tulloch $\mathrm{C}$. Stability of the palatal rugae as landmarks for analysis of dental casts. Angle Orthod 1995;65(1):42-48.

2. Ashmore JL, Kurland BF, King GJ, WheelerTT, Ghafari J, Ramsay DS. A 3-dimensional analysis of molar movement during headgear treatment. Am J Orthod Dentofacial Orthop 2002;121:18-29.

3. Bailey LT, Esmailnejad A, Almeida MA. Stability of the palatal rugae as landmarks for analysis of dental casts in extraction and nonextraction cases. Angle Orthod 1996;66:73-78.

4. Bell A, Ayoub AF, Siebert P. Assessment of the accuracy of a three-dimensional imaging system for archiving dental study models. J Orthod 2003;30:219-223.

5. Bookstein FL. Applying landmark methods to biological outline data. In Mardia KV, Gill CA, Dryden II editors. Image fusion and shape variability. Leeds United Kingdom. University of Leeds Press. 1966:59-70.

6. Bookstein FL. Landmark methods for forms without landmarks: localizinggroup differences in outline shape. Med Image Anal 1997:225-243.

7. Bookstein FL. Size and shape spaces for landmark data in two dimensions. Statistical Sciences 1986;1(2):181242.

8. Cevidanes LH, et al. Superimposition of 3D conebeam CT models of orthognathic surgery patients. Dentomaxillofac Radiol 2005;34:369-375.

9. Cevidanes LH, Styner MA, Proffit WR. Image analysis and superimposition of 3-dimensional cone-beam computed tomography models. Am J Orthod Dentofacial Orthop 2006;129:611-618.

10. Cevidanes LHC, Motta A, Proffit WR, Ackerman JL, Styynere M. Cranial base Superimposition for 3-dimensional evaluation of soft-tissue changes. Am J Orthod Dentofacial Orthop 2010;137:120-129.

11. Cevidanes LHC, Figueiredo Oliveira AE, Grauer D, Styner M, Proffit WR. Clinical Application of 3D Imaging for Assessment of Treatment Outcomes. Seminars in Orthodontics 2011;17(1):72-80.

12. Cha BK, Lee JY, Jost-Brinkmann PG, Yoshida N. Analysis of tooth movement in extraction cases using three-dimensional reverse engineering technology. Eur J Orthod 2007;29:325-331.

13. Champagne M. Reliability of measurements from photocopies of study models. J Clin Orthod 1992;26:648650.

14. Choi JI, Cha BK, Jost-Brinkmann PG, Choi DS, Jang IS. Validity of palatal superimposition of 3-dimensional digital models in cases treated with rapid maxillary expansion and maxillary protraction headgear. Korean J Orthod 2012;42:235-241.

15. Dryden and Mardia Statistical Shape Analysis. Chapters 1,2,3,4. 1998. 
16. Faure J, Treil J, Borianne Ph, Casteigt J, Baron P. Céphalométrie tridimensionnelle en orthopédie dento-faciale. Les possibilités actuelles du logiciel Cépha 3Dt. Orthod Fr 2002;74:19-37.

17. Faure J, Baron P, Treil J. Analyse céphalométrique tridimensionnelle: diagnostic des dysmorphies antéro-postérieures et verticales. Orthod Fr 2005;76(2):91110.

18. Faure J, Marshal-Sixou Ch, Dah-Jouonzo H, Oueiss A, Baron P. Analyse céphalométrique tridimensionnelle à 14 points: compensation des dysmorphies maxillo-faciales. Orthod Fr 2008.79/251-261.

19. Faure J, Oueiss A, Marchal-Sixou Ch, Braga J, Treil J. Céphalométrie tridimensionnelle: applications en clinique et en recherche (Rapport du Congrès SFODFSIDO Venise). Orthod Fr 2008;79:13-30.

20. Faure J, Oueiss A, Treil J, Braga J. Étude en morphométrie géométrique des asymétries faciales: rapports avec les formes basicrâniennes et les pathologies maxillo-faciales ou occlusales. Rev Orthop Dento Faciale 2013;47:155-171.

21. Hoefert CS, et al. Implementing a Superimposition and Measurement Model for 3D Sagittal of Therapyinduced Changes in Facial Soft Tissue: a Pilot Study. J Orofac Orthop 2010;3:221-234.

22. Hoggan BR, Sadowsky C. The use of palatal rugae for the assessment of anteroposterior tooth movements. Am J Orthod Dentofacial Orthop 2001;119:482-8.

23. Jang I, et al. A novel method for the assessment of three-dimensional tooth movement during orthodontic treatment. Angle Orthod 2009;79:447-453.

24. Kau CH, et al. The 3-Dimensional Construction of the Average 11-Year-Old Child Face: A Clinical Evaluation and Application. J Oral Maxillofac Surg 2006; 64:10861092.

25. Kau CH, Richmond S, Incaprera AK, English J, Kia JJ. Three dimensional surface acquisition systems for the study of facial morphology and their application to maxillofacial surgery. Int J Med Robotics Comput Assist Surg 2007;3:97-110.

26. Kau CH, Richmond S. Three Diiemnsional analysis of facial morphology surface changes in untreated children from 12 to 14 years of age. Am J Orthod Dentofacial Orthop 2008;134:751-760.

27. $\mathrm{Kau} \mathrm{CH}$, et al. Use of 3-dimensional surface acquisition to study facial morphology in 5populations. Am J Orthod Dentofaical Orthop 2010;137: S56.e1-S56.e9.

28. Kau CH. Creation of the virtual patient for the study of facial morphology. Facial Plast Surg Clin North Am 2011;19(4):615-622.

29. Keating AP, Knox J, Bibb R, Zhurov Al. A comparison of plaster, digital and reconstructed study model accuracy. J Orthod 2008;35:191-201.

30. Lagravere $\mathrm{MO}$, et al. Reliability of traditional cephalometric landmarks as seen in three dimensional analysis in maxillary expansion treatments. Angle Orthod 2009;79(6):1047-1056.

31. Luebbert J, Ghoneima A, Lagravere MO. Effets squelettiques et dentaires de I'expansion maxillaire rapide évaluée par l'imagerie tridimensionnelle: une étude multicentrique. International Orthodontics 2016;14:1531.

32. MaalTJ, Plooij JM, Rangel FA, Mullemans W, Schutyser FA, Berge SJ. The accuracy of matching three dimensional photographs with skin surfaces derived from cone beam computed tomography. Int J Maxillofac Surg 2008;37:641-646. 
33. Morenhout BA, Gelaude F, Swennen GR, Casselman JW, Van der Sloten J, Mommaerts MY. Accuracy and repeatability of cone beam computed tomography (CBCT) measurements used in the determination of facial indices in the laboratory setup. J CranioMaxillofacial Surg 2009;37:18-23.

34. Nanda $V$, et al. Quantitative analysis of 3-dimensional facial soft tissue photographic images: technical methods and clinical application. Progress in Orthodontics 2015;16:21.

35. Olszewski R, Tanesy O, Cosnard G, Reychler H. Reproductibility of osseous landmarks used for computed tomography based three-dimensional cephalometric analyses. J Craniomaxillofac Surg 2010;38(3):214-221.

36. Oueiss $A$. Les rapports tridimensionnels de la base du crâne et du massif maxillofacial: intérêt en Orthodontie et en Anthropologie. Thèse DU.8/04/2010.UPS Toulouse III.

37. Oueiss A, Pages C, Treil J, Braga J, Baron P, Faure J. Étude des asymétries dans les grandes dysmorphies antéro-postérieures. Orthotod Fr 2010;81:235-244.

38. Raghav P, Suri R, Reddy M, Phull T, Rana T, Jain S. Stability of the palatine rugae: an adjunct to forensic identification. J Dent Health Sci 2015;2(1):39-48.

39. Rossini G, Parrini S, Castroflorio T, Deregibus A, Debernardid CL. Diagnostic accuracy and measurement sensitivity of digital models for orthodontic purposes: A systematic review. Am J of Orthd Dentofacial Orthop 2016;49(2):161-170.

40. Seagara DC, et al. Morphology of an adult Egyptian Population and an adult Houstonian White population Compared Using 3D Imaging. Angle Orthod 2009;79:991-999.

41. Simmons JD, Moore RN, Erickson LC. A longitudinal study of anteroposterior growth changes in the palatine rugae. J Dent Res 1987;66:1512-1515.

42. Treil J, Casteigt J, Escude B, Roch P, Puech C. Intérêt de l'imagerie 3D dans le bilan pré-chirurgical des dysmorphoses maxillo-faciales. Radiologie J CEPUR 1992;12:20-25.

43. Treil J, Casteigt J, Jaeger M, Cavezian R. La charpente maxillo-andibulaire: nouvelle approche cranio-faciométrique tridimensionnelle. Actual Odontostomatol 1993;188627-37.

44. Treil J, Casteigt J, Roch P, Puech C, Cavezian R. Imagerie 3D des dysmorphoses maxillo-faciales. Orthod Fr 1994;64:295-308.

45. Treil J, Casteigt J, Madrid C, Borianne Ph. Une nouvelle construction céphalométrique tridimensionnelle. Un nouveau paramétrage d'analyse tridimensionnelle: les axes d'inertie. Un nouveau concept de l'équilibre maxillo-facial. Orthod Fr 1997;68:171-181.

46. Treil J, Faure J, Braga J, Casteigt J, Borianne Ph. Imagerie and céphalométrie tridimensionnelles des asymétries cranio-faciales. Orthod Fr 2002;73:179-197.

47. Treil J, Casteigt, Faure J, Horn A, Horn A, Labadie M-P. Orthodontie et tomodensitométrie. Rev Othop Dento Faciale 2003;37:9-21.

48. Zamora N, Llamas JM, Cibrin R, Gandia JL, Paredes V. A study of reproducibility of cephalometric landmarks when undertaking a three dimensional (3D) cephalometric analysis. Med Oral Patol Oral Cir Bucal 2012;17(4): 678-688. 